\title{
La degradación ambiental de la cuenca endorreica Coy Aike, Patagonia Austral, Argentina, y su implicancia económica en establecimientos agropecuarios ${ }^{1}$
}

\author{
Environmental degradation in Coy Aike endorheic basin, Austral \\ Patagonia Argentina, and its economic implication in farms
}

\author{
Paula Diez ${ }^{2}$ \\ Mirian Vázquez ${ }^{3}$ \\ Daniel Grima ${ }^{4}$
}

\section{Resumen}

Este trabajo determina los procesos de degradación de la unidad ecológica Matorral de Mata Negra y la disminución de la capacidad de carga producida por la transformación del paisaje en la cuenca endorreica Coy Aike. A partir de la interpretación visual y el análisis de distintos cortes temporales de imágenes satelitales, se caracterizaron cuatro sectores en la cuenca: Laguna, Mata Negra, Vegetación Subarbustiva y Sin Vegetación, siendo los últimos los que presentan mayores evidencias de erosión. La dinámica de la Laguna registró una variación espacial y temporal directamente relacionada con las condiciones meteorológicas, sin embargo, la lengua de erosión asociada no presentó variabilidad. Estas últimas afectan la vegetación natural por la disminución de la disponibilidad de tierras aptas para pastoreo, y ocasionan pérdidas en el valor textil de la lana. El cálculo del costo económico que produce la erosión de los suelos se determinó teniendo en cuenta la receptividad ovina media por hectárea para el área

1 Proyecto de investigación código A29/291, financiado y otorgado por UNPA. "Caracterización ecosistémica y económica de los humedales en el sur de Santa Cruz, Patagonia Argentina”. Director: Mirian Vázquez, codirector: Paula Diez.

2 Doctor en Geografía, Universidad Nacional del Sur. Asistente de docencia de la Universidad Nacional de la Patagonia Austral, Unidad Académica Río Gallegos. Argentina. pau.diez@hotmail.com

3 Geógrafo de la Universidad de la Patagonia Austral. Docente de la Universidad Nacional de la Patagonia Austral, Unidad Académica Río Gallegos. Argentina. mirian.vazquez@gmail.com

4 Ingeniero en Recursos Naturales Renovables de la Universidad de la Patagonia Austral. Ayudante de docencia de la Universidad Nacional de la Patagonia Austral, Unidad Académica Río Gallegos. Argentina. dgrima@speedy.com.ar 
ecológica. En el establecimiento Coy Aikem, las 211 ha de tierra improductiva representan al productor una pérdida económica de 1.925 dólares anuales; no obstante, la gravedad económica estará dada por la cantidad de bajos con potencialidad erosiva, que existan en los establecimientos agropecuarios.

Palabras clave: cuenca endorreica, degradación, paisaje, Patagonia, Argentina.

\begin{abstract}
This work determines the degradation processes of the ecological unit of Mata Negra scrub and decreasing load produced by transformation of the landscape in Coy Aike endorheic basin. Visual interpretation using several satellite images, characterizing four sectors in the basin: Lagoon of Mata Negra, Sub scrub vegetation and without vegetation, the last mentioned with the highest erosion evidence. The dynamics of the lagoon showed a spatial and temporal variation directly related to weather conditions, but the lagoon does not contain variability associated with erosion. The last ones affect natural vegetation decreasing availability of land suitable for grazing and losses in the commercial value of the wool for textile. The estimates of economic costs that cause soil erosion was determined taking into account the average per ovine hectare receptivity to the ecological area. In establishing the 211 hectares of Coy Aike unproductive lands must represent an economic loss to the producer of USD 1,925.00 per year, however the economic gravity will coincide with the amount of basin and with the erosive potential that exist in agricultural establishments.
\end{abstract}

Keywords: endorheic basin-degradation-landscape-Patagonia Argentina. 


\section{Introducción}

El paisaje, por su fisonomía, es una entidad reconocible y diferenciable de otras vecinas. Urban et al., (1987) consideran los paisajes terrestres como un "mosaico heterogéneo de formas del relieve, tipos de vegetación y usos de la tierra”. Leser y Rood (1991) definen el paisaje como un "complejo de patrones físicos, bióticos y antropogénicos directa $\mathrm{o}$ indirectamente interrelacionados entre sí, formando una correlación funcional". Desde un enfoque más general, el paisaje es una representación espacial del ecosistema, un área geográfica donde se plasman estudios ecológicos. Zonneveld (1995) define el paisaje como la "unidad mínima cartografiable que permite indicar espacialmente los principales componentes de un ecosistema".

El paisaje se transforma por diversos procesos que se suceden de manera ordenada y se superponen parcialmente. La secuencia de transformación comienza con el proceso de perforación seguido por el de disección, fragmentación y achicamiento. La perforación representa el establecimiento de elementos aislados, con límites netos en una matriz homogénea. La disección, como segundo paso, aparece como un corte de la matriz con líneas de ancho constante. El proceso se completa con la fragmentación o ruptura de la matriz en parches del ecosistema, de tamaño y forma variable, y, por último, el achicamiento produce la reducción gradual de los parches hasta su desaparición (Forman, 1995; Hunter, 1996; Matteucci, 1998). Las principales causas de dicho proceso están asociadas a efectos derivados de acciones antrópicas como la expansión urbana, la industrialización, la agricultura intensiva y los fenómenos de crecimiento de la infraestructura viaria. La ampliación de las redes de comunicación es una de las causas de la fragmentación, no tanto por la pérdida de superficie neta sino por la ruptura del funcionamiento del conjunto del territorio.

Oliva et al., (2001) delimitan en la provincia de Santa Cruz, Argentina, ocho áreas ecológicas, zonificadas a partir de la metodología FAO (1997) que integra características físicas de la tierra con factores socio-económicos y un amplio rango de uso de tierras. En el presente trabajo se determinan los procesos de degradación de la unidad ecológica Matorral de Mata Negra (MMN) y la disminución de la capacidad de carga producida por la transformación del paisaje en la cuenca endorreica Coy Aike.

El área ecológica MMN se ubica al sureste de la provincia de Santa Cruz y posee un clima que se cataloga entre árido y semiárido. Es una estepa arbustiva de porte medio, dominada en un 60 a $70 \%$ por la mata negra (Junielia tridens), en algunos casos en forma continua y en otros en forma de mosaicos de estepa graminosa de coirón amargo (Stipa speciosa) o coirón blanco (Festuca pallescens), según reportan Cuadra y Oliva (1994). Las cuencas endorreicas forman parte del $17 \%$ de humedales de zonas áridas de Argentina (Verbist et. al., 2010), según la definición adoptada 
por la Convención de los Humedales de Importancia Internacional o Convención Ramsar (Canevari et al., 1998), y para Patagonia constituyen un valioso recurso natural, pues ofrecen pasturas y agua para el desarrollo de actividades agropecuarias, principalmente la ganadería.

La escasez hídrica de las zonas áridas y semiáridas, agravada por las sequías periódicas que allí ocurren y la intervención antrópica de los ecosistemas, desencadena procesos de erosión del suelo, que modifican los elementos biofísicos y socioculturales de una región, y que se traduce en pérdida de biodiversidad y productividad, y, en consecuencia, en la transformación del paisaje (FAO 1979; FAO 1994; CNEH \& CAACH, 2003). Según FAO (2001), "la calidad del uso de la tierra no es un valor absoluto, pero deben ser evaluadas con relación a las funciones de la tierra y al uso específico que se pretende hacer de ella".

Por este motivo, es de suma importancia comprender la dinámica de los procesos de transformación del paisaje, para describir cuantitativamente los fenómenos espaciales, predecir la evolución temporal y espacial, y analizar el efecto que genera la propagación de las perturbaciones, ya sea en un marco de conservación o de planificación del uso de los elementos del paisaje (Matteucci, 1998). Los objetivos de este trabajo incluyeron identificar y caracterizar los distintos sectores de la cuenca endorreica Coy Aike, analizar la dinámica espacial y temporal de la laguna y la pluma eólica asociada, y examinar el costo económi- co que produce la degradación del suelo para un establecimiento agropecuario.

\section{1. Área de estudio}

La cuenca endorreica Coy Aike presenta un cuerpo lacustre de tamaño variable en su interior. Está ubicado al sureste de la provincia de Santa Cruz, Patagonia Argentina ( $51^{\circ} 04^{\prime} 13,9^{\prime}$ ' LS y $69^{\circ} 32^{\prime}$ $03,7^{\prime}$ ' LO), en la zona de contacto entre la meseta sedimentaria y la terraza más elevada del valle del río Coyle, en una región sin drenaje definido entre los ríos Santa Cruz y Coyle (Caballero, 2000). Posee una extensión de 1.660 ha y forma parte de las 93.484 ha del establecimiento agropecuario homónimo (Figura 1). El clima de la región está definido por valores medios anuales de temperatura del orden de los $6{ }^{\circ} \mathrm{C}$ y precipitación de $240 \mathrm{~mm}$. El viento es el elemento característico de la región, constante a lo largo del año, predominantemente del sector oeste, y se manifiesta con mayor rigor durante los meses más cálidos. Las ráfagas alcanzan los $140 \mathrm{~km} \mathrm{~h}^{-1} \mathrm{e}$ intensifican las condiciones de sequedad del ambiente (Mazzoni et al., 2002).

La cuenca, de pendiente suave (1\%), se encuentra disectada en sentido norte-sur por la ruta nacional número tres, dejando en el sector oeste la cubeta, los cordones de gravas depositados por la acción del oleaje durante eventos de tormenta (Mazzoni et al., 2001) y la mayoría de red de cañadones que la drenan. $\mathrm{Al}$ este se delimita la pluma eólica origi- 


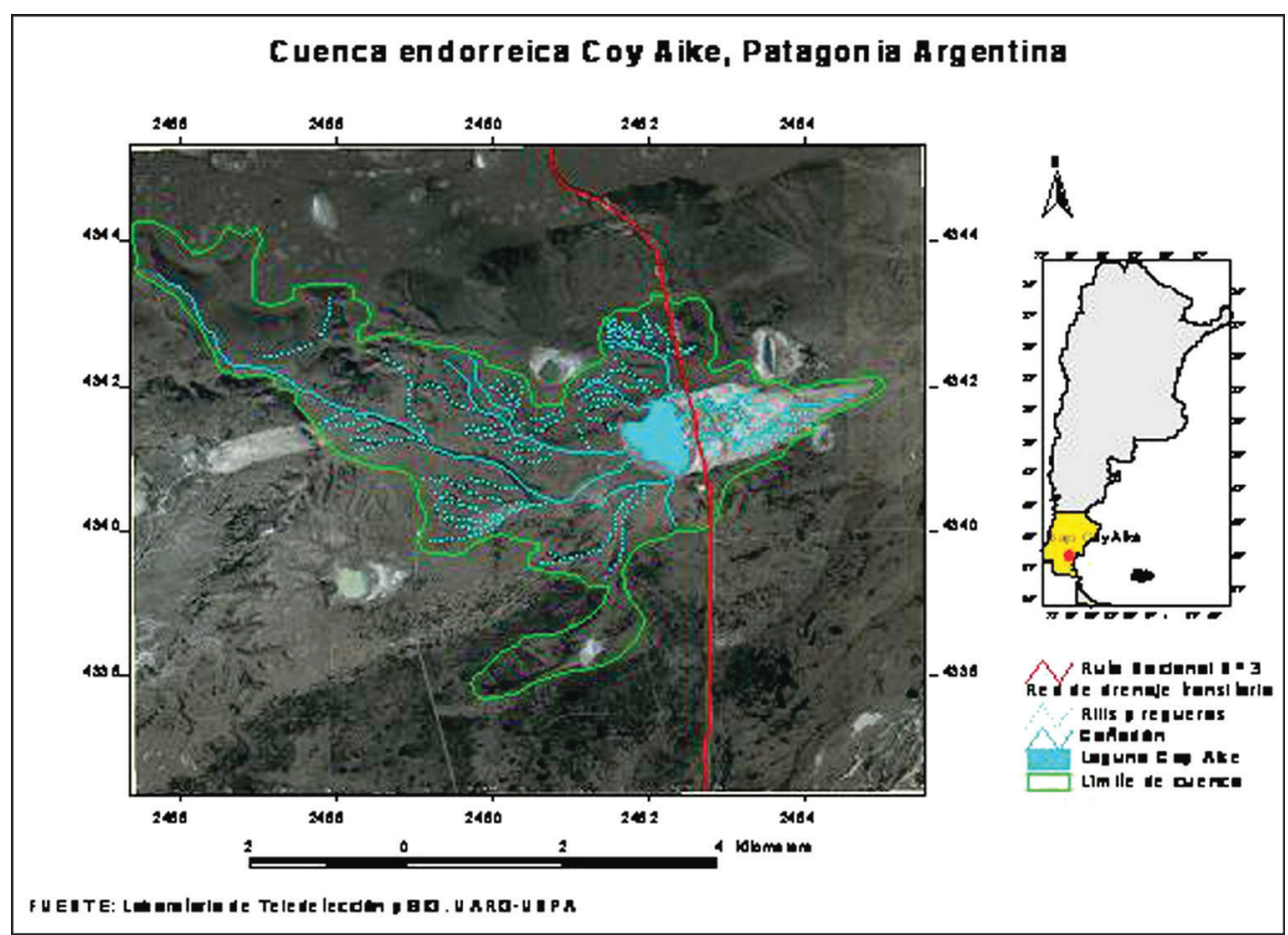

Figura 1. Mapa ubicación del bajo Coy Aike.

nada por procesos de degradación eólica e hídrica principalmente (FAO 1979;

FAO 1994; Oldeman et al., 1990).

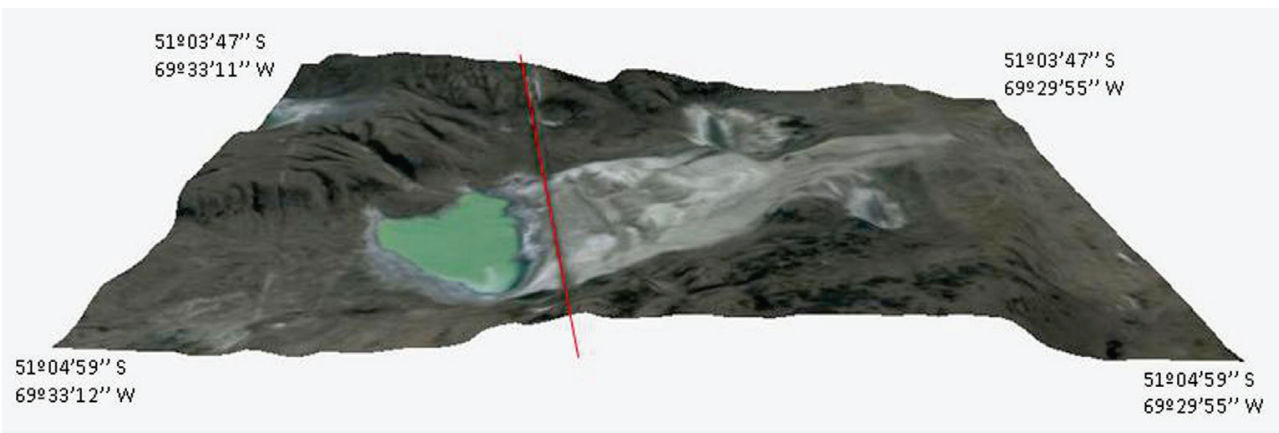

Figura 2a. Modelo digital de elevación de la cuenca Coy Aike.

Fuente: JPL-SRTM-NASA (2000). 


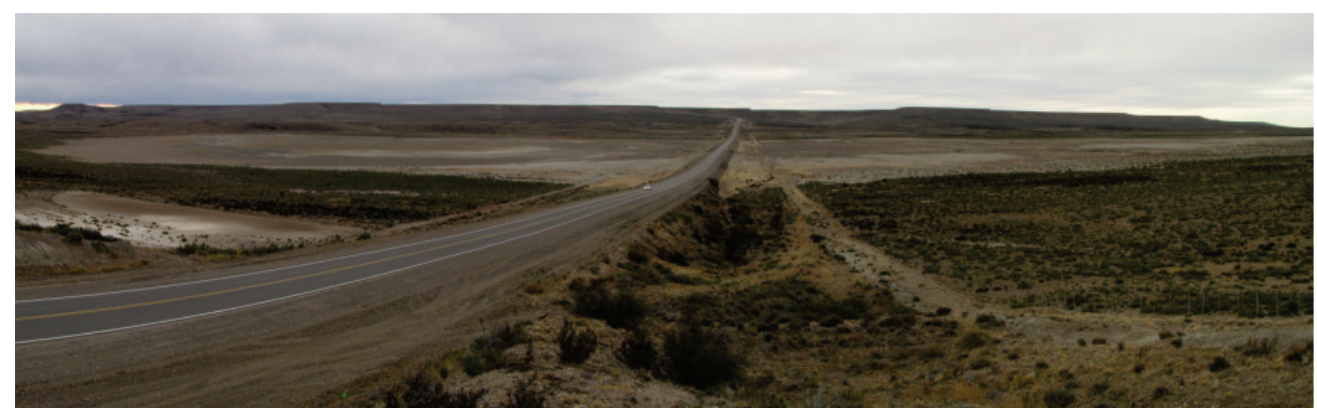

Figura 2b. Vista panorámica de la cuenca

Fuente: Paula Diez

\section{Metodología}

La identificación de los distintos parches de la cuenca endorreica Coy Aike se realizó a partir de la interpretación visual de la imagen multiespectral de alta resolución QuickBird $(2,44 \mathrm{~m})$ del año 2003. Este tipo de interpretación se basa en el uso de patrones de forma, textura, tamaños y topológicos entre objetos para discriminar las unidades que se interpretan. La imagen georreferenciada (Faja 2 Gauss Krugger, Elipsoide de referencia WGS 84), fue utilizada como apoyo para las distintas capas de información temática, motivo por el cual los atributos espaciales, cálculo de las superficies de cada parche, corresponden al momento de toma de la imagen.

Una característica importante desde el punto de vista del crecimiento vegetal, es la salinidad y el $\mathrm{pH}$ de los suelos. Con el fin de determinar esta correlación, se obtuvieron muestras de suelo en cada uno de los parches identificados. Estas muestras se secaron al aire y se tamizaron por malla plástica cuadrada de $2 \mathrm{~mm}$ (ASTM $\mathrm{n}^{\circ} 18$ ). La salinidad queda evidenciada mediante la conductividad eléctrica, la cual se hizo con un conductímetro Horiba, con celda copa de constante $=0,75$. La conductividad eléctrica y el $\mathrm{pH}$ se efectuaron sobre la pasta de suelo saturada. Las determinaciones de $\mathrm{pH}$ se llevaron a cabo con un pHmetro Horiba H24 y electrodo de vidrio combinado. La distribución granulométrica de los suelos se determinó por el método de la pipeta. Para facilitar la dispersión de las muestras, se utilizó una solución de hexametafosfato de sodio y carbonato de sodio. Posteriormente, se estableció la clase textural de acuerdo con el sistema USDA, Soil Conservation Service (1983).

Para analizar la dinámica del cuerpo de agua y evaluar la evolución de la pluma eólica, se seleccionaron las imágenes Landsat ETM 229-96 (Convenio CONAE-UNPA) de distintos cortes temporales entre los años 1986 y 2006. La superficie de la laguna se calculó a partir de la digitalización en pantalla. El análisis se completó con la elaboración 
de perfiles espaciales, en sentido oesteeste, para comprobar el cambio de altura de la lámina de agua a partir de los valores de reflectancia de la banda 1 de las imágenes Landsat TM de 1986 y ETM de 2002. La banda $1(0,45$ a 0,52 micrones, azul) está diseñada para penetrar en cuerpos de agua y diferenciar entre suelo y vegetación (Chuvieco, 1998).

El cálculo del costo económico que produce la erosión de los suelos se realizó teniendo en cuenta la receptividad ovina media por hectárea para el área ecológica. Según el Task Group on Unity in Concepts and Terminology (1995), "la receptividad o carrying capacity es el número promedio de animales domésticos y/o silvestres que pueden ser mantenidos en una unidad de manejo". Esta varía, en consecuencia, con la composición fisonómico florística de las áreas ecológicas consideradas. Así, la receptividad para el MMN es de 0,24 EO ha. Equivalente ovino (EO) es la unidad que permite comparar cargas de animales de distintas categorías o diferentes estados fisiológicos y corresponde al requerimiento energético de una oveja de 49 $\mathrm{kg}$ que desteta un cordero a los cien días $(2,45$ Mcal de energía metabolizable por animal y por día).

Además se consideró el rendimiento al lavado, definido como el peso de lana limpia (libre de suarda, tierra y cualquier otro material que se remueva con el lavado), que puede ser obtenido de una unidad de masa de lana sucia (Rodríguez , 1998). Este rendimiento se expresa en porcentaje.
Los valores calculados se refieren al establecimiento agropecuario Coy Aike, por ser este el que contiene la cuenca considerada en el análisis. De las 93.484 ha de superficie total, $1.660 h a$ corresponden a la cuenca endorreica; el cálculo de productividad se hizo sobre el sector de 211 ha correspondiente a los parches: sin vegetación y con vegetación subarbustiva de cobertura muy escasa.

La rentabilidad estimada anual de los campos es el producto de la superficie del campo por la receptividad. Para su cálculo se consideró el precio que se obtendría por la venta de cordero y de lana considerando el valor de mercado para la temporada 2011 (sitio web oficial del PROLANA).

\section{Resultados y discusión}

\subsection{Identificación y caracterización de parches en la cuenca endorreica Coy Aike}

Dentro del área de estudio se identificaron cuatro macroparches: Laguna, Mata Negra, Vegetación Subarbustiva y Sin Vegetación (Figura 3). La ruta nacional número tres tiene un rol importante dentro del sistema, ya que atraviesa el área en sentido norte-sur, dejando principalmente el macroparche Laguna y Mata Negra al oeste, y el parche Sin Vegetación y el dominado Vegetación Subarbustiva, al este. Se tomaron en total siete muestras de suelo a ambos lados de la ruta, cubriendo todos los 
parches identificados. La Tabla 1 indica los lugares de muestreo. Sólo el punto de muestreo L-5 se obtuvo al oeste de la ruta, en el parche Laguna, el resto de las muestras se extrajo del sector este, correspondiendo la muestra P-1 a la Mata Negra, los puntos P-2 y P-3 al parche Vegetación Subarbustiva sectores sur y norte respectivamente, y P-4, P6 y P7 a distintos sectores del parche Sin Vegetación.

En general, los suelos muestreados son de reacción leve a medianamente alcalina, con excepción de la muestra P-1, que presentó un pH neutro. Los valores de conductividad eléctrica evidencian poca salinidad, con la salvedad de la muestra
L-5, que mostró una fuerte salinidad, y L-7, que posee salinidad extrema.

Los suelos son de textura variada. Los porcentajes para cada una de las fracciones granulométricas del sistema del United State Department of Agriculture se presentan en la Tabla 2.

La ruta nacional número tres atraviesa la cuenca en sentido norte-sur; su construcción previó la instalación de una alcantarilla que permite la interacción de ambos sectores del paisaje. En el interior de la misma se pudo observar que los sedimentos finos transportados por el agua vuelven al sector de la laguna para recomenzar el ciclo (Figura 4).

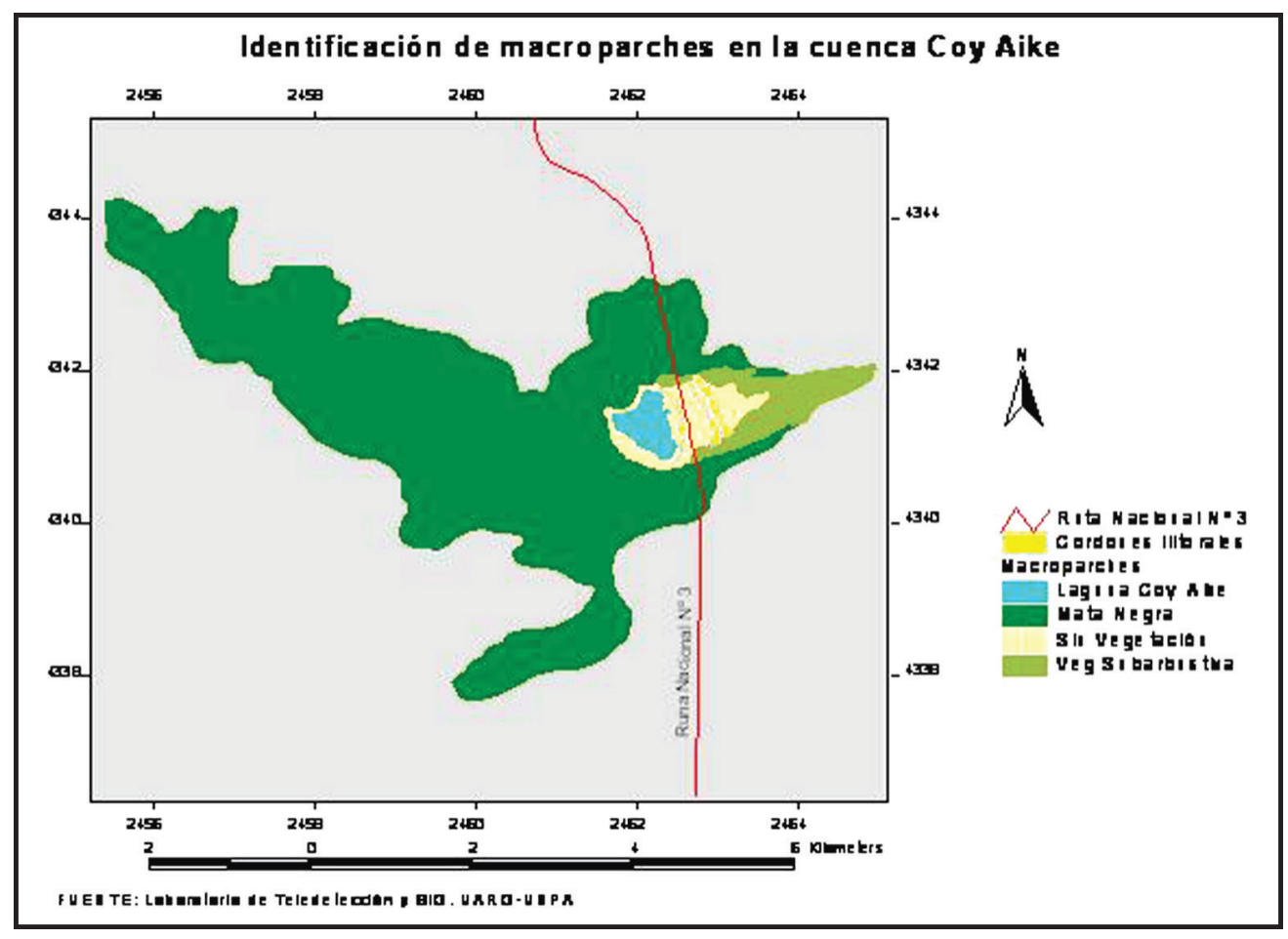

Figura 3. Identificación de parches en el bajo Coy Aike. 
Tabla 1. Valores de pH y conductividad eléctrica de los suelos.

\begin{tabular}{|c|c|c|}
\hline Muestra & $\mathbf{p H}$ & $\begin{array}{l}\text { Conductividad } \\
\qquad\left(\mathrm{dS} \mathrm{m} \mathbf{m}^{-1}\right)\end{array}$ \\
\hline P-1 (P. de Mata Negra) & 7,05 & 0,215 \\
\hline P-2 (P. Veg. Subarbustiva s ector sur) & 8,22 & 3,176 \\
\hline P-3 (P. Veg. Subarbustiva sector norte) & 8.51 & 6,559 \\
\hline P-4 (P. Sin Vegetación) & 8,81 & 4,136 \\
\hline L-5 (P. Laguna) & 9,08 & 9,551 \\
\hline L-6 (P. Sin Vegetación sector cordón litoral) & 9,18 & 4,954 \\
\hline
\end{tabular}

Tabla 2. Composición granulométrica de las muestras de suelo, clasificación USDA.

\begin{tabular}{l|r|r|r|r|r|r|r}
\hline $\begin{array}{c}\text { Mracción } \\
(\%)\end{array}$ & P-1 & P-2 & P-3 & P-4 & L-5 & L-6 & L-7 \\
\hline Arcilla & 6,17 & 20,74 & 25,93 & 59,26 & 64,66 & 26,06 & 18,30 \\
\hline Limo total & 15,81 & 11,78 & 19,34 & 20,40 & 15,49 & 8,38 & 6,04 \\
\hline Limo fino & 8,96 & 6,95 & 11,75 & 11,72 & 10,22 & 4,56 & 3,74 \\
\hline Limo grueso & 6,85 & 4,83 & 7,59 & 8,68 & 5,26 & 3,82 & 2,30 \\
\hline Arena total & 78,02 & 67,48 & 54,73 & 20,34 & 19,86 & 65,56 & 75,66 \\
\hline A. muy fina & 17,45 & 8,67 & 9,42 & 4,72 & 8,44 & 6,62 & 8,08 \\
\hline A. fina & 28,53 & 35,44 & 27,77 & 7,38 & 8,13 & 24,92 & 40,06 \\
\hline A. media & 16,32 & 13,14 & 11,39 & 4,00 & 1,10 & 16,72 & 21,39 \\
\hline A. gruesa & 11,41 & 4,97 & 4,63 & 2,67 & 1,49 & 10,88 & 6,07 \\
\hline A. muy gruesa & 4,31 & 5,26 & 1,52 & 1,57 & 0,69 & 6,42 & 0,06 \\
\hline Clase textural USDA & AFr & FrA & FArA & Ar & Ar & FArA & FrA \\
\hline
\end{tabular}

Donde: (A) arenoso, (Fr) franco, (Ar) arcilloso 

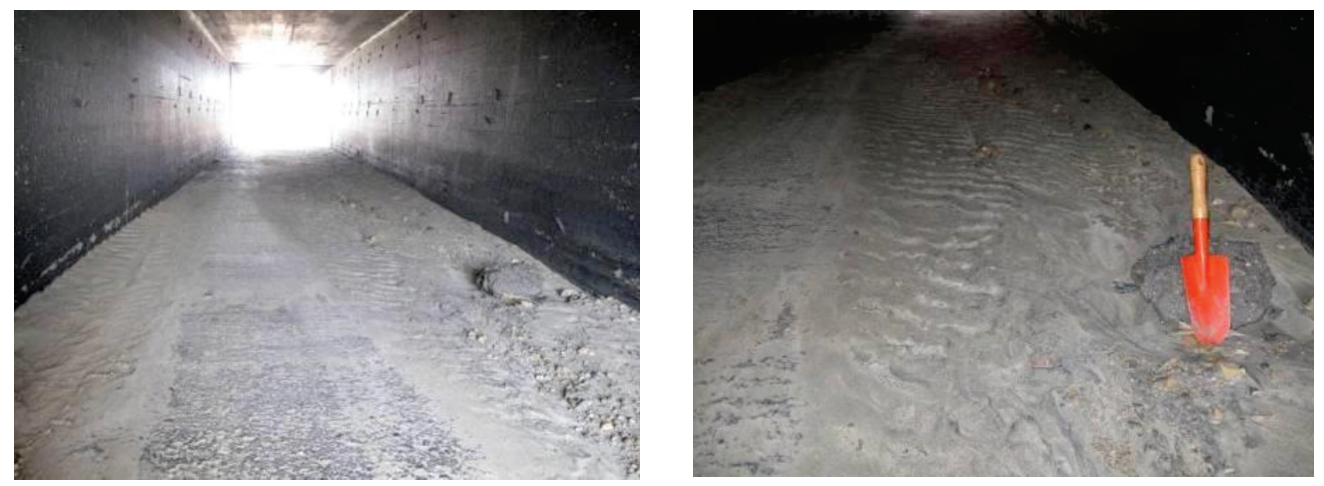

Figura 4. Alcantarilla en la ruta nacional número tres. Obsérvense los sedimentos finos producto del transporte hídrico y el diseño de la microtopografía que indica la dirección del flujo.

Macroparche de la Laguna Coy Aike: corresponde a la cubeta o fondo de la depresión, cuya cota es de $50 \mathrm{msnm}$ aproximadamente. Al momento de la toma de la imagen (año 2003), presenta una extensión de $45 h a$, y en la actualidad se encuentra apenas con una pequeña lámina de agua (Figura 5a). Muestra una superficie suavemente cóncava donde se acumula agua estacionalmente. Los años de escasa precipitación determinaron la desaparición total de la laguna, generando un área de deflación donde

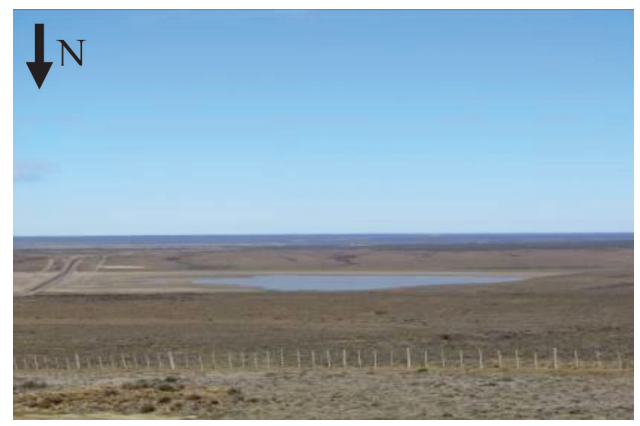

Figura 5a. Vista de la cubeta con una pequeña lámina de agua, en agosto de 2011. los procesos erosivos desencadenados dejan las partículas de suelo disponibles para el transporte. A medida que el agua se evapora, los materiales del fondo se secan y agrietan y pueden ser fácilmente transportados por el viento, dejando expuesto un sustrato arcilloso $(64,66 \%)$ que hace imposible la infiltración del agua (Figura 5b). Los valores de conductividad eléctrica en L-5, evidencian la alta salinidad del fondo de la cubeta $\left(9,551 \mathrm{dS} \mathrm{m}^{-1}\right)$.

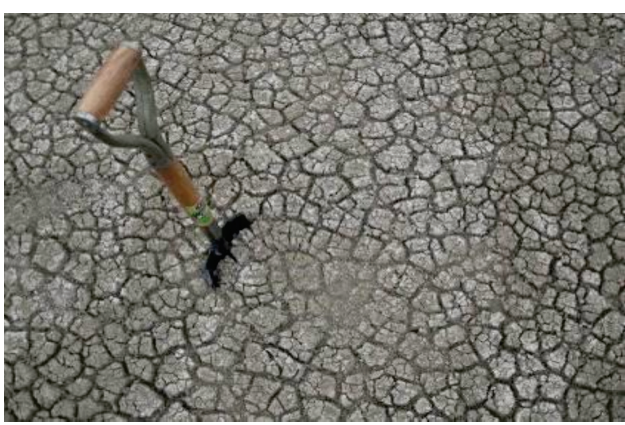

Figura 5b. Obsérvense las grietas de desecación del fondo de la cubeta, en febrero de 2011. 
Parche de Vegetación de Mata Negra (Junellia tridens): es el de mayor extensión de la cuenca con 1.404 ha. Se corresponde a la unidad ecológica MMN, en que los suelos son predominantemente Aridisoles y Molisoles de textura arenosa y buen drenaje por presentar abundantes rodados en todo el perfil (Oliva et al., 2001). La muestra analizada indicó un $78 \%$ de arena, en su mayoría fina a medias y con baja salinidad, correspondiendo a una clase textural arenosa franca. Es una estepa arbustiva de porte medio, de unos $70 \mathrm{~cm}$ de altura, dominada en un 60-70 \% por la mata negra, en algunos casos de forma continua y en otros en forma de mosaicos de estepa graminosa de coirón fueguino (Festuca gracillima), aunque también pueden ser comunes los coirones amargos (Stipa speciosa, Stipa chysophylla). En el estrato de las gramíneas bajas se reconocen coirón poa (Poa dusenii), coirón enano (Stipa ibari), coirón pluma (Stipa neaei), Festuca pyrogea y Rytidosperma virescens (Figura 6).

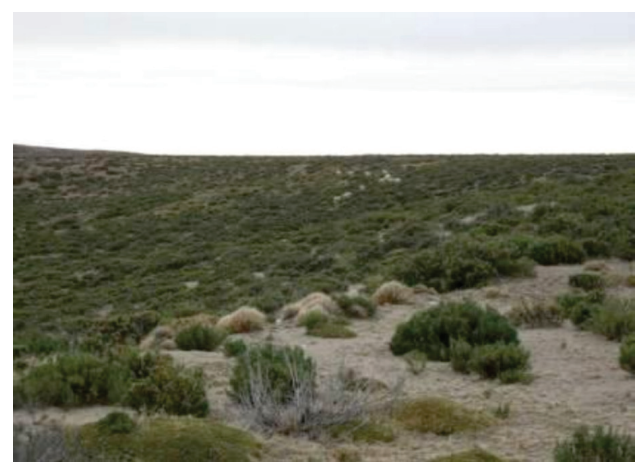

Figura 6. Fisonomía vegetal del parche Mata Negra.

Parche de Vegetación Subarbustiva: corresponde a la zona de contacto entre el parche de Vegetación Mata Negra y el parche Sin Vegetación. Es una superficie de 109 ha aproximadamente, y cubre las laderas de la cuenca en el sector más oriental de la misma (Figura 7a). La dirección predominante del viento determina que el material suelto, producto de la desecación de la laguna, sea transportado y entrampado en los arbustos. El suelo es principalmente franco arenoso al sur y de características más arcillosas, al norte. El parche adquiere una geomorfología de dunas de un metro cuadrado de superficie aproximadamente, que rodean y cubren la vegetación impidiendo su normal desarrollo y provocando la muerte paulatina de las mismas (Figura 7b).

En los sectores donde la vegetación permanece viva, se observan rasgos de degradación física, como madrigueras y compactación del suelo, causados por la presencia de hábitat de roedores y pisoteo de animales de mayor porte (Figura 8). 


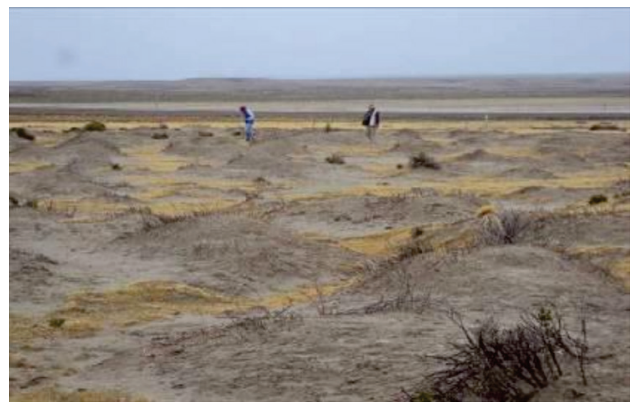

Figura 7a. Panorama general del parche.

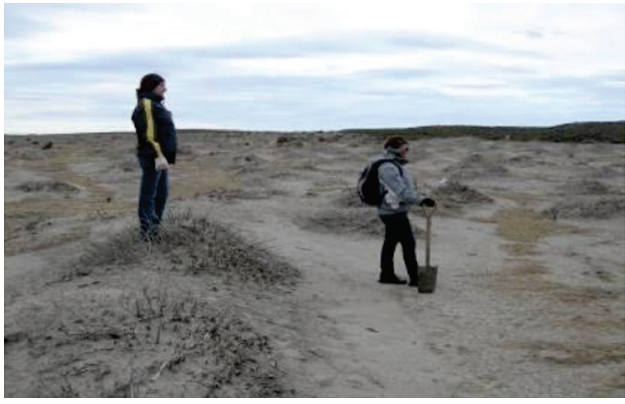

Figura 7b. Vista de las dunas de arcilla. Nótese la diferencia de altura entre los montículos de arcilla entrampados en la vegetación y la base del parche.

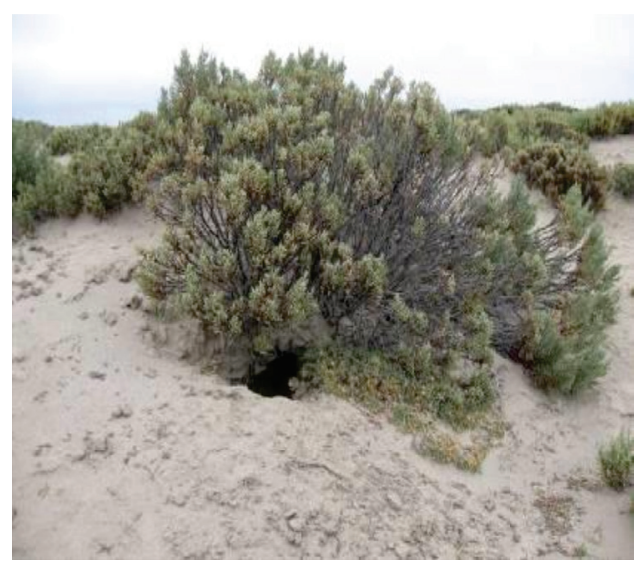

Figura 8. Madriguera socavada en Mata Negra.

Parche Sin Vegetación: tiene una superficie aproximada de $102 \mathrm{ha}$. Al oeste de la ruta se encuentra rodeando a la cubeta una geoforma de playa de acumulación (Mazzoni et al., 2001) constituida por una capa de gravas en forma de cordones depositados por la acción del oleaje durante eventos de tormenta (Figura 9a). $\mathrm{Al}$ este se extiende la pluma de erosión donde se observa una acción combinada de procesos de erosión eólica e hídrica. El primero dio lugar a la formación de pavimentos de desierto, en los cuales la acción del viento remueve las partículas finas del suelo y solo se conservan expuestos los fragmentos de mayor tamaño como gravas y guijarros (Figura $9 b$ ). Sobre estos, con dirección este a oeste siguiendo el sentido de la pendiente, se evidencian procesos de erosión hídrica. Este proceso genera una intrincada red de rills, cárcavas y surcos de carácter efímero que transportan nuevamente los sedimentos más finos, que no fueron movidos por el viento, hacia el sector de la laguna (Figura 10). 
Este sector es el más degradado de la cuenca, por ser el más afectado por los agentes erosivos. Tiene una gran cárcava central que muestra la gran fragilidad del terreno, que en su mayoría es de textura arcillosa. Al ser escasa la cobertura vegetal, las gotas de lluvia impactan en la superficie y saturan rápidamente la capacidad de infiltración del suelo. El

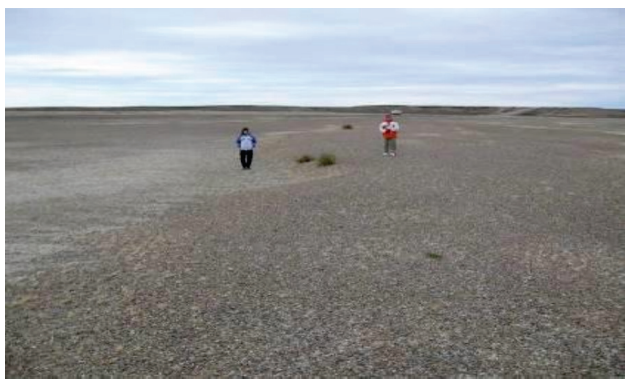

Figura 9a. Vista del cordón litoral de gravas que rodea la cubeta.

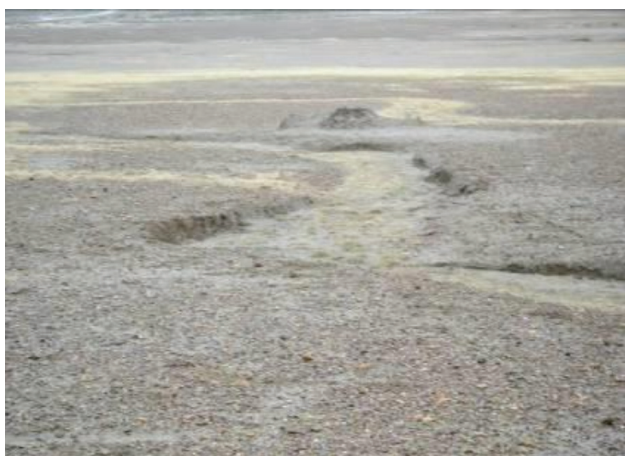

agua comienza a escurrir rápidamente provocando cambios importantes en el paisaje después de cada precipitación. El lavado de las laderas por escurrimiento en manto es significativo y en sectores con pendientes moderadas a fuertes, puede eliminar la delgada capa fértil del suelo.

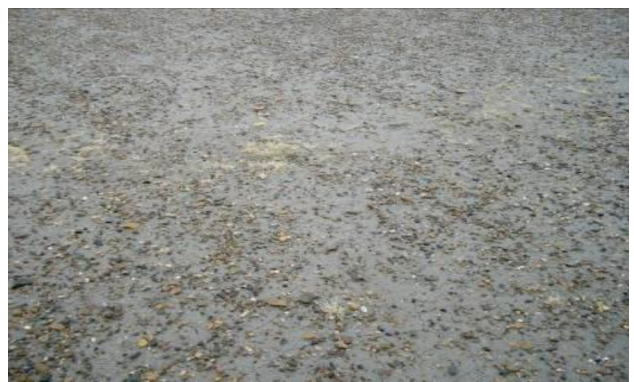

Figura 9b. Detalle del pavimento de desierto.

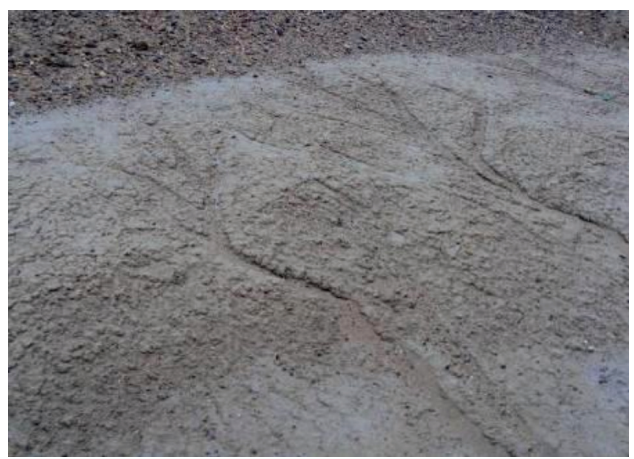

Figura 10. Surcos y rills generados por erosión hídrica.

En cercanía de la banquina de la ruta nacional número tres, la fisonomía del parche cambia. Aparece un sustrato de textura arenosa encostrado y se observan acumulaciones de arena suelta sobre la base de los surcos y cañadones (Figura 11).

\subsection{Dinámica espacial y temporal de la laguna y la pluma eólica asociada}

Las lagunas de régimen temporario ubicadas al sur de la provincia de Santa Cruz, suelen secarse en los años de escasa precipitación o durante el verano 


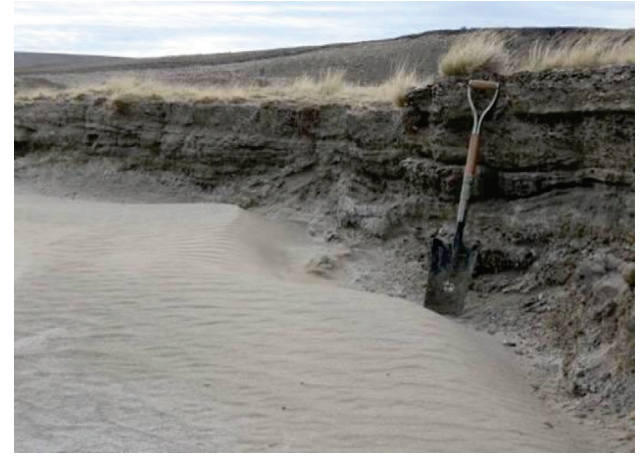

cuando la evaporación se ve favorecida por los intensos y frecuentes vientos estacionales (Mazzoni, et al., 1999, 2002). La laguna de la cuenca Coy Aike corresponde a este grupo, por lo cual registra una variación espacial y temporal directamente relacionada con las condiciones meteorológicas de la región. Está alimentada por nueve cañadones principales que, junto a otros de menor importancia, rills y regueros, son los responsables de recolectar las precipitaciones que alimentan la laguna (Figura 1).

El primer corte temporal se hizo en el año 1986, momento en que la laguna presentó una superficie de 13,16 ha. En adelante y hasta el año 2002, el aumento de la superficie de la laguna fue progresivo. En 1999 mostró cerca del doble de la superficie anotada una década antes $(23,18 h a)$. Para el año 2001, llegó a 46,28 ha, registrando el máximo alcanzado en el año 2002 con 70,02 ha (Figura 12).

Este valor máximo de superficie se corresponde con los datos meteorológicos establecidos para la región. En el año

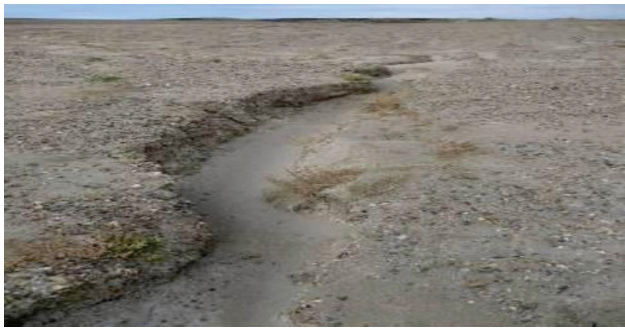

Figura 11. Perfil de suelo arenoso encostrado y detalle de las acumulaciones de arena en la base de los surcos.

2002, la precipitación media anual de $417 \mathrm{~mm}$ superó ampliamente los 240 mm de precipitación media histórica. Los cortes temporales siguientes, muestran el comienzo del proceso de desecación del cuerpo de agua. En el año 2004, la superficie era de 68,78 ha y para el año 2006 se había reducido a 47,32 ha, situación similar a la del año 2001 (Tabla 3).

Una medición hecha a partir de una imagen de Google Earth del año 2008, mostró que la laguna continuaba disminuyendo $(45,07 h a)$. En los años siguientes (2009 y 2010), la laguna desapareció completamente. Este dato se obtuvo de observación directa en campo. De igual forma se determinó que a comienzos de 2011 presentó una pequeña lámina de agua, resultado de las lluvias ocurridas recientemente en la región y la profundización de las cárcavas (Figura 13).

Los perfiles espaciales realizados para dos cortes temporales, muestran claramente la variación de la cantidad de agua de la laguna a través de los valores de reflectancia. Mayor valor del píxel indica menor profundidad del pelo de 
Tabla 3. Variabilidad de la superficie de la laguna

\begin{tabular}{r|rrrrrr}
\hline Año & 1986 & 1999 & 2001 & 2002 & 2004 & 2006 \\
\hline Área (ha) & 13,16 & 23,18 & 46,28 & 70,02 & 68,78 & 47,32 \\
\hline
\end{tabular}
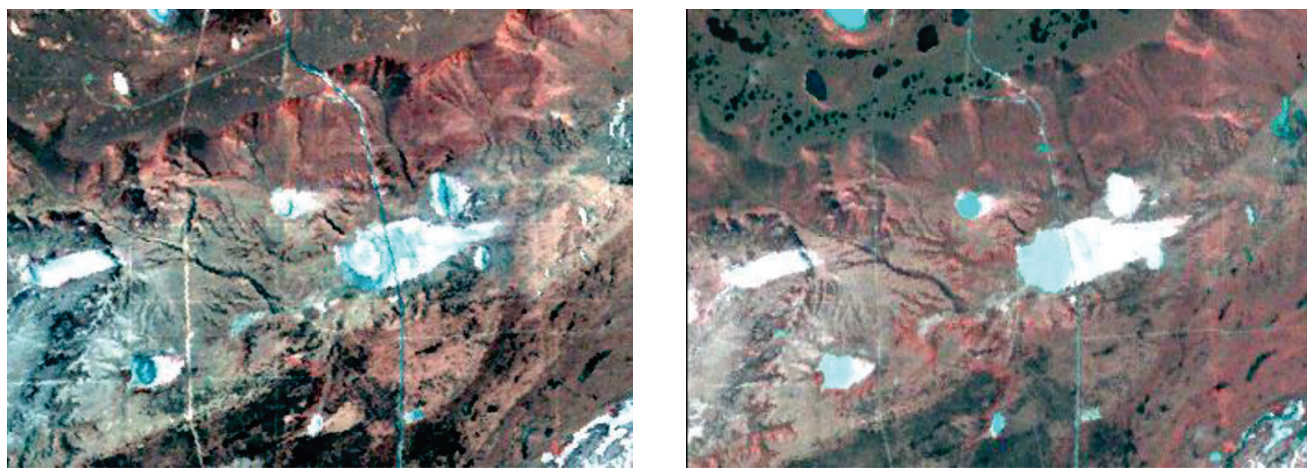

Figura 12. Laguna Coy Aike en distintos períodos. Las imágenes satelitales Landsat TM de febrero de 1986 (a) y la ETM de septiembre de 2002 (b), muestran la diferencia en la disponibilidad de agua de la cuenca.

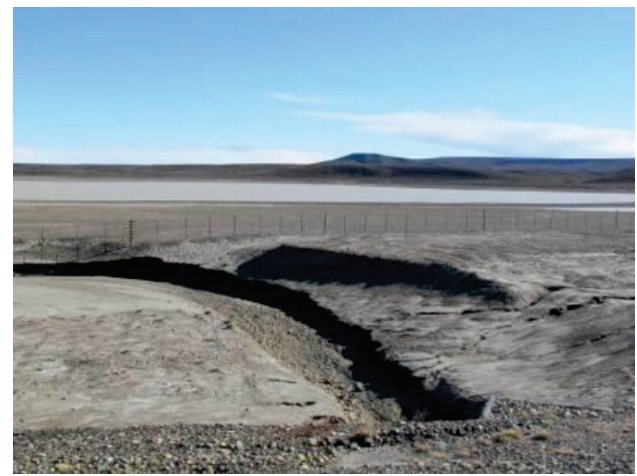

Figura 13. Situación de la laguna en febrero de 2011.

agua y viceversa (Figura 14). En el perfil efectuado en la imagen del año 1986, los primeros $400 \mathrm{~m}$ corresponden a superficie de suelo seco; a partir de los $500 \mathrm{~m}$, los altos y bajos en los valores de reflectancia pertenecen a sectores con presencia y ausencia de agua. El pico descendente que aparece pasados los $1000 \mathrm{~m}$, corresponde a la ruta. La poca profundidad del agua de la laguna en ese período, muestra valores de reflectancia de 120, información que concuerda con los datos meteorológicos para ese tiempo $(16,1 \mathrm{~mm}$ de precipitación en febrero de 1986).

El perfil de septiembre de 2002 presenta un comportamiento totalmente diferen- 
te. El espejo de agua aparece de manera continua respondiendo una cobertura total de agua en esa superficie. El perfil muestra menor cantidad de suelo seco, lo que indica una mayor superficie de la laguna. Los valores de píxel son mucho más bajos en general; en el sector del suelo seco, el valor es inferior a 40, resultado de la humedad contenida en el mismo. En cuanto a los valores corres- pondientes a la laguna, cercanos a 60 , señalan una mayor profundidad de esta. En diciembre de 2002, el perfil muestra la misma forma que el del mes de septiembre del mismo año pero con valores más altos de reflectancia. Esto es resultado de la alta evaporación que sufre la región a raíz de los intensos vientos estacionales.
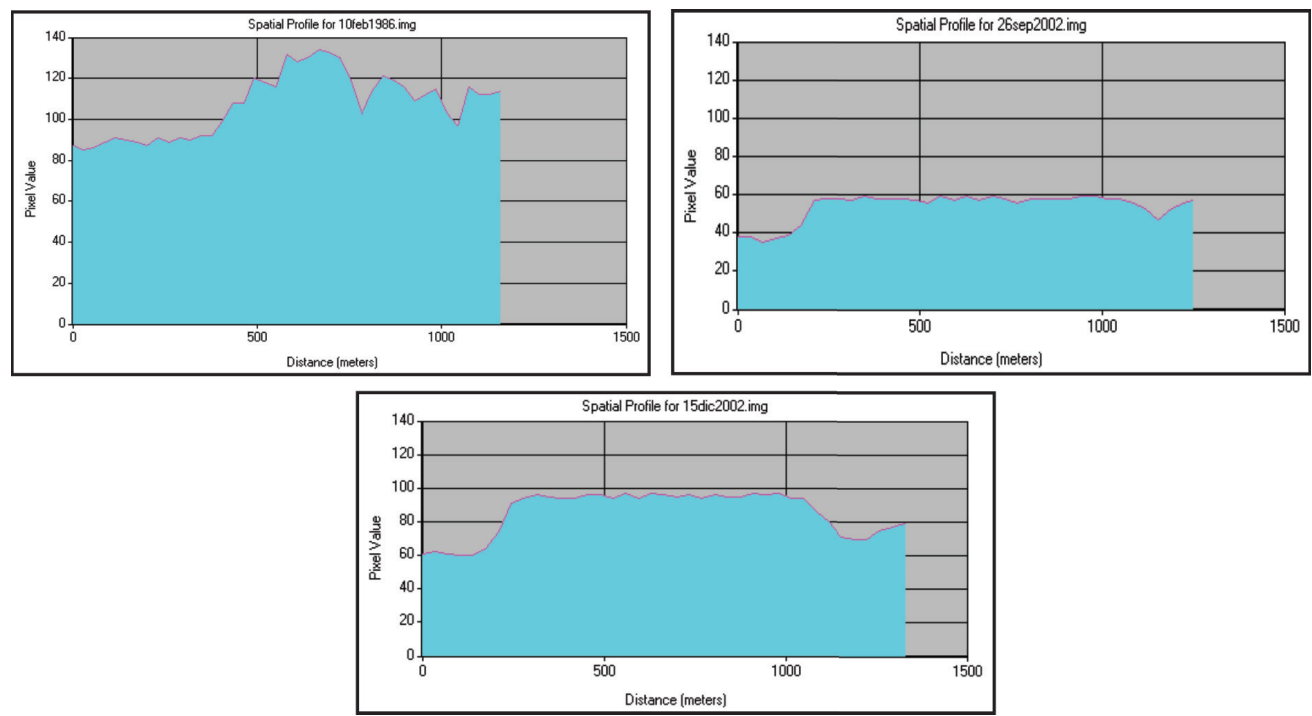

Figura 14. Variación de cantidad de agua en la laguna Coy Aike en distintos cortes temporales. Febrero 1986, septiembre 2002 y diciembre 2002.

La laguna Coy Aike presenta una pluma eólica activa donde alternan procesos de deflación y acumulación. A través de los cortes temporales se observó que solo en el año 1999 el tamaño de la misma varió. En 1986, la longitud de la pluma era de $2,26 \mathrm{~km}$ y para el año 1999 , de $1,1 \mathrm{~km}$. En los siguientes cortes (2001, 2002, 2004 y 2006), la pluma mantuvo su tamaño en alrededor de 2,5 km (Tabla 4).

Tabla 4. Dinámica de la pluma eólica

\begin{tabular}{r|rrrrrr}
\hline Año & 1986 & 1999 & 2001 & 2002 & 2004 & 2006 \\
\hline Longitud (km) & 2,26 & 1,1 & 2,5 & 2,4 & 2,4 & 2,5 \\
\hline
\end{tabular}


3.3 Análisis del costo económico producido por la erosión de los suelos de la cuenca endorreica para el establecimiento agropecuario Coy Aike

A medida que las plumas eólicas extienden su longitud afectando la vegetación natural, disminuye la disponibilidad de tierras aptas para el uso agropecuario, principal actividad económica de la región. Sumado a la pérdida de superficie destinada a pastoreo, el avance de las plumas eólicas genera también pérdidas en el valor textil de la lana, específicamente en el rendimiento al lavado. Los sedimentos finos que conforman la estructura de las plumas eólicas, quedan retenidos en el vellón aumentando el porcentaje de lana sucia y por ende disminuyendo su valor económico.

Los sectores improductivos se traducen en una disminución en la renta. Las 211 ha de pluma eólica le generan al productor una pérdida económica de 1.925 dólares anuales. Este valor corresponde a lo que se esperaría ganar de la venta de lana y corderos, si la pluma no existiera (Tabla 5).

Tabla 5. Cálculo económico de la venta de lana y corderos, en dólares.

\begin{tabular}{|c|c|c|}
\hline \multicolumn{3}{|l|}{ VENTA DE LANA } \\
\hline Detalle & Valores Unitario & Valor Final \\
\hline Superficie afectada (ha) & 211,00 & \\
\hline Receptividad estimada (EO/ha) & 0,38 & \\
\hline Numero de EO - Ovejas & & 79,8 \\
\hline Peso promedio de vellón socio en $\mathrm{kg}$ & 4,30 & \\
\hline Peso promedio de vellón limpio en $\mathrm{kg}$ & 2,75 & \\
\hline Total de lana limpia en $\mathrm{kg}$ & & 343,1 \\
\hline Valor de mercado (27 micrones) dólares de EE.UU. & 2,37 & \\
\hline Valor total dólares de EE.UU. & & 813,2 \\
\hline \multicolumn{3}{|l|}{ VENTA DE CORDEROS } \\
\hline Detalle & Valores Unitarios & Valor total \\
\hline Porcentaje de señalada & 70 & 55.9 \\
\hline Venta de corderos en frigorífico (\%). & 50 & 27.9 \\
\hline Peso promedio en $\mathrm{kg}$ & 10 & 279.3 \\
\hline Valor de mercado temporada 2011 (dólares de EE.UU. & 3,98 & $1.111,6$ \\
\hline
\end{tabular}

Los principales rasgos geomórficos que alteran la homogeneidad del paisaje son las cuencas endorreicas que perforan la matriz del área ecológica MMN, constituyendo puntos de alta fragilidad am- biental. Este primer paso de transformación se da de manera natural, ya que los bajos se formaron principalmente por acción eólica e hidroeólica (Fidalgo, 1972; Rabassa, et al., 1984). Estas geo- 
formas de dimensiones variables suelen contener cuerpos

lacustres o salinas en su interior, que ocasionalmente favorecen el crecimiento de pequeños mallines de vegetación halófita (Mazzoni \& Vázquez, 2004).

Sin embargo, cuando esos cuerpos de agua tienen escaso nivel o están secos, la base de las depresiones constituye una fuente de materiales para el transporte eólico, lo cual favorece el desarrollo de lenguas de erosión (Movia, 1984) o "plumas eólicas" (Mazzoni, 2001), las cuales van migrando paulatinamente siguiendo la dirección predominante del viento.

La secuencia de transformación del paisaje en Coy Aike continuó con la traza de la ruta nacional número tres, que fragmentó por la mitad a la cuenca endorreica. Si bien aparece en el paisaje como un elemento que altera la dinámica del ecosistema, no representa la causa de los procesos erosivos. La construcción de la ruta previó la instalación de una alcantarilla que permite la interacción de ambos sectores del paisaje.

A medida que los procesos de erosión del suelo extienden su longitud, afectan la vegetación natural y disminuyen la disponibilidad de tierras aptas para el uso agropecuario, como la ganadería ovina extensiva, principal actividad económica de la región (Vazquez et al., 2008).

\section{Conclusiones}

Los humedales de régimen temporario actúan como concentradores de las escasas precipitaciones que caen en la región. La presencia de agua, la disponibilidad de vegetación halófita y el reparo ante las inclemencias de tiempo por la diferencia de altura con respecto a la meseta circundante, contribuyen a que el ganado utilice estos espacios de manera intensiva, favoreciendo la degradación de la vegetación y el incremento de los procesos erosivos.

El manejo sustentable de los pastizales naturales depende en gran medida de la conservación de los suelos. La principal causa de pérdida irreversible de la productividad en Patagonia es la erosión de los suelos por sobrepastoreo. Tal vez, este fue el inicio de los procesos erosivos en la cuenca Coy Aike, los que se agravaron posteriormente por la acción conjunta del viento y el agua.

La pérdida económica quizás no sea significativa, pero hay que tener en cuenta que estas depresiones son un rasgo típico en el paisaje patagónico austral, y la gravedad económica está dada por la cantidad de bajos con potencialidad erosiva que existan en cada establecimiento agropecuario. Según Borelli (2001), desde una visión regional, puede esperarse que los establecimientos rurales localizados en la misma área presenten características productivas y problemas similares. 


\section{Literatura citada}

Borrelli, P. (2001). Producción animal sobre pastizales naturales. En P. Borrelli \& G. Oliva. Ganadería ovina sustentable en la Patagonia Austral. Tecnología de manejo extensivo. Buenos Aires: INTA.

Caballero, J. (2000). Recursos hídricos superficiales. En A. García \& E. Mazzoni. El gran libro de la Provincia de Santa Cruz. Madrid: Alfa Centro Literario, Millenium.

Canevari, P., Blanco, D., Bucher, E., Castro, G. \& Davidson, I. (1998). Los humedales de la Argentina. Clasificación, situación actual, conservación y legislación. Buenos Aires: Wetlands International-Américas, Publicación 46, 208 p.

Centro Neotropical de Entrenamiento en Humedales, CNEH y Corporación Ambientes Acuáticos de Chile, CAACH. (2003). Alianza mundial para la conservación de los humedales en las zonas áridas y desertificadas: una tarea impostergable. Chile: Centro Neotropical de Entrenamiento en Humedales, Corporación Ambientes Acuáticos de Chile. Recuperado de http://sad.dga.cl

Cuadra, D. \& Oliva, G. (1994). Ambientes naturales de la provincia de Santa Cruz. Revista Espacios de la Universidad Nacional de la Patagonia Austral, UNPA, $2,(6), 25-35$.

Chuvieco, E. (1996). Fundamentos de teledetección espacial. ( $3^{a}$ ed.). Madrid: Rialp, 568 p.

FAO. (1997). Zonificación agro-ecológica. Dirección de Fomento de Tierras y Aguas. Boletín 73. Roma: FAO.

FAO. (1979). A provisional methodology for soil degradation assessment. Roma: FAO.

FAO. (1994) Land degradation in South Asia: its severity, causes and effects upon the people. World Soil Resources Report 78. Roma: FAO.

FAO. (2001). Indicadores de la calidad de la tierra y su uso para agricultura sostenible y desarrollo rural. Boletín de Tierras y Aguas de la FAO 5. Roma: Banco Mundial, PNUMA, PNUD, FAO.

Fidalgo, F. (1972). Consideraciones sobre los bajos situados al norte de la provincia de Santa Cruz. En: V Congreso Geológico Argentino, celebrado en Córdoba. Actas 5, 123-137. 
Forman, R. T. (1995). Some general principles of landscape and regional ecology. Landscape Ecology 10, 133-142.

Hunter, H.J. (1996). Fundamentals of Conservation Biology. s.l: Blackwell Scientific Publications

JPL-SRTM-NASA. (2000). Jet Propulsion Laboratory, Shuttle Radar Topography Mission, National Aeronautics and Space Administration. Retrieved from http://www2.jpl.nasa.gov/srtm/

Leser, H. \& Rood, H. (1991). Landscape ecology: fundamentals, aims and perspective. In: G. Esser \& D. Overdieck (eds.). Modern Ecology. Basic and applied Aspects. (pp. 831-844). Amsterdam. Elsevier.

Matteucci, S. (1998). El análisis regional desde la ecología. En: Matteucci \& Buzai. Sistemas ambientales complejos: herramientas de análisis espacial 5. (pp. 117150). EUDEBA. Buenos Aires.

Mazzoni, E., Vázquez, M., Ferrari, S., Belardi, J., Albrieu, C., Carballo, F., Espinosa, S., Larrosa, J. \& Gismondi, D. (1999, diciembre). Las cuencas endorreicas o "bajos sin salida": consideraciones acerca del estudio de una de las geoformas típicas del paisaje patagónico. Revista Espacios, 5 (16), 45-50. Secretaría de Extensión de Rectorado de la Universidad Nacional de la Patagonia Austral. Río Gallegos.

Mazzoni E. (2001). Distribución espacial y caracterización geomorfológica de pequeñas cuencas endorreicas de la Patagonia austral extracordillerana. Anales del Instituto de la Patagonia, Serie Ciencias Naturales XXIX, 5-23. UMAG, Chile.

Mazzoni, E., Vázquez, M. \& Ruiz, E. (2002). Dinámica geomorfológica de depósitos eólicos asociados a "bajos sin salida" de la Patagonia austral. Actas IV Jornadas de Geografía Física, 165, 176. Mar del Plata.

Mazzoni, E., Ruiz, E., Vázquez, M., Baetti, C. \& Martínez, G. (2001). Unidades de paisaje en pequeñas cuencas endorreicas de la Patagonia extracordillerana argentina. Anales de la Sociedad Chilena de Ciencias Geográficas, 95-102. Talca, Chile.

Mazzoni, E. \& Vázquez, M. (2004). Ecosistemas de mallines y paisajes de la Patagonia Austral (Provincia de Santa Cruz). Río Gallegos. INTA. 64 pp.

Movia, C. (1972). Formas de erosión eólica en la Patagonia (Argentina). Photointerpretation $n^{0} 6 / 3$. Paris, Francia: Technit. 
Oldeman, L.R., Hakkeling, R.T.A. \& Sombroek, W.G. (1990). World map of human-induced soil degradation. Wageninen: ISRIC, UNEP.

Oliva, G., González L., Rial, P. \& Livraghi, E. (2001). Áreas ecológicas de Santa Cruz y Tierra del Fuego. En P. Borrelli \& G. Oliva. Ganadería ovina sustentable en la Patagonia Austral. Tecnología de manejo extensivo. Buenos Aires: INTA.

PROLANA, Secretaría de Ganadería, Agricultura y Pesca. Presidencia de la Nación. (s.f.). Recuperado de www.sgp.gov.ar

Rabassa, J., Brandani, A., Capua, O. \& Ottonello, E. (1984). Curso de campo en sistemas ecogeomorfológicos: guía de excursiones. Neuquén, Argentina: Departamento de Postgrado, Universidad Nacional del Comahue.

Rodríguez, R. M. (1998). Producción ovina. Departamento de Agronomía. Universidad Nacional del Sur. Rev. 30.

Task Group on Unity in Concepts an Terminlogy Society for Range Management. (1995). New concepts for assessment of rangeland condition. J. Range Manage, $48,271-282$.

Urban, D.L., O'Neill, R.V . \& Shugart, H.H. (1987). Landscape Ecology. BioScience 37, 284-307.

USDA - Soil Conservation Service (1983). Principles and procedures for using soil survey laboratory data. (Prepared by Stajj of the National Soil Survey Laboratory). Washington D.C.: Lourse avide. 131 p.

Vázquez, M., Grima, D. \& Mazzoni, E. (2008). SIG para la evaluación y monitoreo de pequeñas cuencas endorreicas en la Patagonia Austral. Universidad de Santa Maria, Rio Grande do Sul. Brasil. Revista Geografía, Ensino \& Pesquiza, 12(1), 1608-1620.

Verbist, K., Santibáñez, F., Gabriels, D. \& Soto, G. (2010). Atlas de zonas áridas de América Latina y El Caribe. CAZALAC. Documentos Técnicos del PHILAC.

Zonneveld, I.S. (1995). Land Ecology, an Introduction to Landscape Ecology as base for Land Evaluation, Land management and Conservation. Amsterdam: SPB Academic Publishing. 
Recepción: 29 de noviembre de 2012

Evaluación: 23 de enero de 2013

Aprobación: 20 de abril de 2013 